\title{
OUTPUT REgULATION OF THE SIMPLIFIED LORENZ CHAOTIC SYSTEM
}

\author{
Sundarapandian Vaidyanathan ${ }^{1}$ \\ ${ }^{1}$ Research and Development Centre, Vel Tech Dr. RR \& Dr. SR Technical University \\ Avadi, Chennai-600 062, Tamil Nadu, INDIA \\ sundarvtu@gmail.com
}

\begin{abstract}
This paper investigates the problem of designing feedback controllers for regulating the output of the simplified Lorenz system, which is a novel chaotic system recently discovered by K.H. Sun and J.C. Sprott (2009). The simplified Lorenz chaotic system is a three-dimensional chaotic system with one bifurcation parameter and it includes the Lorenz attractor (1963) as a special case. In this paper, state feedback control laws to regulate the output of the simplified Lorenz chaotic system have been derived so as to track the constant reference signals. The control laws are derived using the regulator equations of C.I. Byrnes and A. Isidori (1990), who solved the problem of output regulation of nonlinear systems involving neutrally stable exosystem dynamics. Numerical simulations are shown to illustrate the results.
\end{abstract}

\section{KEYWORDS}

Simplified Lorenz, System, Output Regulation, Nonlinear Control Systems, Feedback Stabilization.

\section{INTRODUCTION}

Designing feedback control laws for regulating the output of control systems is one of the very active research problems in control systems theory. For linear control systems, the output regulation problem was solved by Francis and Wonham ([1], 1975). For nonlinear control systems, the output regulation problem was solved by Byrnes and Isidori ([2], 1990). Using Centre Manifold Theory [3], Byrnes and Isidori derived regulator equations, which characterize the solution of the output regulation problem of nonlinear control systems satisfying some stability assumptions.

The output regulation problem for nonlinear control systems has been studied extensively by various scholars in the last two decades [4-14]. In [4], Mahmoud and Khalil obtained results on the asymptotic regulation of minimum phase nonlinear systems using output feedback. In [5], Fridman solved the output regulation problem for nonlinear control systems with delay using centre manifold theory. In [6-7], Chen and Huang obtained results on the robust output regulation for output feedback systems with nonlinear exosystems. In [8], Liu and Huang obtained results on the global robust output regulation problem for lower triangular nonlinear systems with unknown control direction.

In [9], Immonen obtained results on the practical output regulation for bounded linear infinitedimensional state space systems. In [10], Pavlov, Van de Wouw and Nijmeijer obtained results on the global nonlinear output regulation using convergence-based controller design. In [11], Xi and Dong obtained results on the global adaptive output regulation of a class of nonlinear systems with nonlinear exosystems. In [12-14], Serrani, Isidori and Marconi obtained results on the semi- 
global and global output regulation problem for minimum-phase nonlinear systems. In [15-17], we derived output regulation results for some three-dimensional chaotic systems using regulator equations.

In this paper, we solve the output regulation problem for the simplified Lorenz chaotic system ([18], 2009). We derive state feedback control laws solving the constant regulation problem of the simplified Lorenz chaotic system using the regulator equations of Byrnes and Isidori (1990). The simplified Lorenz chaotic system is an important one-parameter family of three-dimensional chaotic systems discovered by Sun and Sprott ([18], 2009). As special cases, the simplified Lorenz chaotic system includes the Lorenz system ([19], 1963). The unified chaotic system has important applications in Electronics and Communication Engineering.

This paper is organized as follows. In Section 2, we provide a review the problem statement of output regulation problem for nonlinear control systems and the regulator equations of Byrnes and Isidori [2], which provide a solution to the output regulation problem under some stability assumptions. In Section 3, we describe the simplified Lorenz chaotic system and its special case. In Section 4, we describe the main results of this paper, namely, the solution of the output regulation problem for the simplified Lorenz chaotic system for the important case of constant reference signals (set-point signals). In Section 5, we describe the numerical results validating and illustrating the effectiveness of the regulation schemes derived in this paper. In Section 6, we conclude with the main results obtained in this paper for the simplified Lorenz system.

\section{Review of the Output Regulation Problem For Nonlinear CONTROL SySTEMS}

In this section, we consider a multi-variable nonlinear control system described by

$$
\begin{aligned}
& \dot{x}=f(x)+g(x) u+p(x) \omega \\
& \dot{\omega}=s(\omega) \\
& e=h(x)-q(\omega)
\end{aligned}
$$

Here, the differential equation (1a) describes the plant dynamics with state $x$ defined in a neighbourhood $X$ of the origin of $R^{n}$ and the input $u$ takes values in $R^{m}$ subject to te effect of a disturbance represented by the vector field $p(x) \omega$. The differential equation (1b) describes an autonomous system, known as the exosystem, defined in a neighbourhood $W$ of the origin of $R^{k}$, which models the class of disturbance and reference signals taken into consideration. The equation (2) defines the error between the actual plant output $h(x) \in R^{p}$ and a reference signal $q(\omega)$, which models the class of disturbance and reference signals taken into consideration.

We also assume that all the constituent mappings o the system (1) and the error equation (2), namely, $f, g, p, s, h$ and $q$ are continuously differentiable mappings vanishing at the origin, i.e.

$$
f(0)=0, g(0)=0, p(0)=0, s(0)=0, h(0)=0 \text { and } q(0)=0 .
$$

Thus, for $u=0$, the composite system (1) has an equilibrium $(x, \omega)=(0,0)$ with zero error (2).

A state feedback controller for the composite system (1) has the form

$$
u=\rho(x, \omega)
$$

where $\rho$ is a continuously differentiable mapping defined on $X \times W$ such that $\rho(0,0)=0$. 
International Journal of Control Theory and Computer Modelling (IJCTCM) Vol.1, No.3, November 2011

Upon substitution of the feedback control law (3) into (1), we get the closed-loop system

$$
\begin{aligned}
& \dot{x}=f(x)+g(x) \rho(x, \omega)+p(x) \omega \\
& \dot{\omega}=s(\omega)
\end{aligned}
$$

\section{State Feedback Regulator Problem [2]:}

Find, if possible, a state feedback control law $u=\rho(x, \omega)$ such that the following conditions are satisfied.

(OR1) [Internal Stability] The equilibrium $x=0$ of the dynamics

$$
\dot{x}=f(x)+g(x) \rho(x, 0)
$$

is locally exponentially stable.

(OR2) [Output Regulation] There exists a neighbourhood $U \subset X \times W$ of $(x, \omega)=(0,0)$ such that for each initial condition $(x(0), \omega(0)) \in U$, the solution $(x(t), \omega(t))$ of the closed-loop system (4) satisfies

$$
\lim _{t \rightarrow \infty}[h(x(t))-q(\omega(t))]=0 .
$$

Byrnes and Isidori [2] solved the output regulation problem stated above under the following two assumptions.

(H1) The exosystem dynamics $\dot{\omega}=s(\omega)$ is neutrally stable at $\omega=0$, i.e. the exosystem is Lyapunov stable in both forward and backward time at $\omega=0$.

(H2) The pair $(f(x), g(x))$ has a stabilizable linear approximation at $x=0$, i.e. if

$$
A=\left[\frac{\partial f}{\partial x}\right]_{x=0} \text { and } B=\left[\frac{\partial g}{\partial x}\right]_{x=0}
$$

then $(A, B)$ is stabilizable.

Next, we recall the solution of the output regulation problem derived by Byrnes and Isidori [2].

Theorem 1. [2] Under the hypotheses (H1) and (H2), the state feedback regulator problem is solvable if and only if there exist continuously differentiable mappings $x=\pi(\omega)$ with $\pi(0)=0$ and $u=\varphi(\omega)$ with $\varphi(0)=0$, both defined in a neighbourhood of $W^{0} \subset W$ of $\omega=0$ such that the following equations (called the regulator equations) are satisfied:

(1) $\frac{\partial \pi}{\partial \omega} s(\omega)=f(\pi(\omega))+g(\pi(\omega)) \varphi(\omega)+p(\pi(\omega)) \omega$

(2) $h(\pi(\omega))-q(\omega)=0$

When the regulator equations (1) and (2) are satisfied, a control law solving the state feedback regulator problem is given by

$$
u=\varphi(\omega)+K[x-\pi(\omega)]
$$

where $K$ is any gain matrix such that $A+B K$ is Hurwitz. 


\section{DESCRIPTION OF THE SIMPLIFIEd LORENZ CHAOTIC SySTEM}

The simplified Lorenz chaotic system ([18], 2009) is described by the dynamics

$$
\begin{aligned}
& \dot{x}_{1}=10\left(x_{2}-x_{1}\right) \\
& \dot{x}_{2}=-x_{1} x_{3}+(24-4 c) x_{1}+c x_{2} \\
& \dot{x}_{3}=x_{1} x_{2}-\frac{8}{3} x_{3}
\end{aligned}
$$

where $x_{1}, x_{2}, x_{3}$ are the state variables and $c$ is real parameter taking values in $[-1.59,7.75]$.

In ([18], 2009), Sun and Sprott proposed the simplified Lorenz chaotic system described in (6) and discussed the chaotic behaviour of the system for various values of $c$. Obviously, the system (6) reduces to the original Lorenz system for $c=-1$.

The state orbits of the simplified Lorenz chaotic system for $c=1$ are shown in Figure 1.

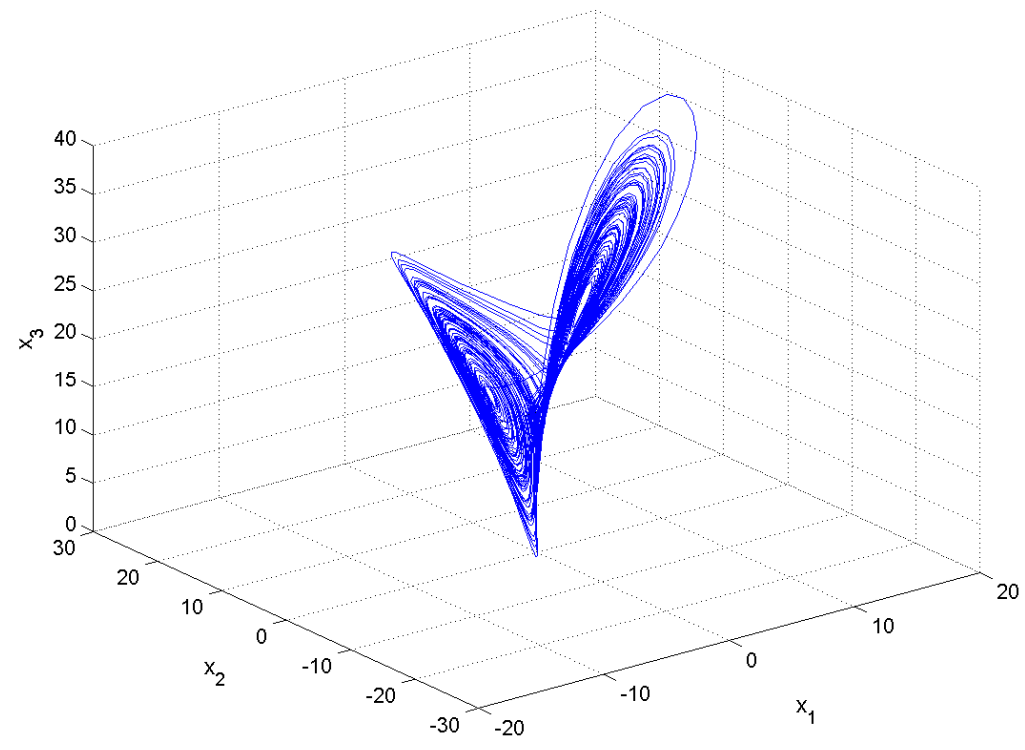

Figure 1. State Orbits of the Simplified Lorenz Chaotic System

\section{OUtPut Regulation OF The Simplified LoRenz ChaOtic System}

In this paper, we solve the output regulation problem for the simplified Lorenz chaotic system ([18], 2009) for the tracking of the constant reference signals (set-point signals).

Thus, we consider the simplified Lorenz chaotic system described by the dynamics

$$
\begin{aligned}
& \dot{x}_{1}=10\left(x_{2}-x_{1}\right) \\
& \dot{x}_{2}=-x_{1} x_{3}+(24-4 c) x_{1}+c x_{2}+u_{1} \\
& \dot{x}_{3}=x_{1} x_{2}-\frac{8}{3} x_{3}
\end{aligned}
$$

where $x_{1}, x_{2}, x_{3}$ are the state variables, $u$ is the control and $c \in[-1.59,7.75]$. 
International Journal of Control Theory and Computer Modelling (IJCTCM) Vol.1, No.3, November 2011

The constant reference signals are generated by the scalar exosystem dynamics

$$
\dot{\omega}=0
$$

It is important to note that the exosystem given by (8) is neutrally stable, because the exosystem (8) admits only constant solutions. Thus, the assumption (H1) of Theorem 1 holds trivially.

Linearizing the dynamics of the simplified Lorenz chaotic system (7) at $x=0$, we obtain

$$
A=\left[\begin{array}{ccc}
-10 & 10 & 0 \\
24-4 c & c & 0 \\
0 & 0 & -8 / 3
\end{array}\right] \quad \text { and } B=\left[\begin{array}{l}
0 \\
1 \\
0
\end{array}\right]
$$

The system pair $(A, B)$ can be expressed as

$$
A=\left[\begin{array}{cc}
A_{1} & 0 \\
0 & \lambda^{*}
\end{array}\right] \text { and } B=\left[\begin{array}{c}
B_{1} \\
0
\end{array}\right],
$$

where

$$
A_{1}=\left[\begin{array}{lr}
-10 & 10 \\
24-4 c & c
\end{array}\right] \text { and } B_{1}=\left[\begin{array}{l}
0 \\
1
\end{array}\right]
$$

It is easy to see that the system pair $\left(A_{1}, B_{1}\right)$ is completely controllable and the uncontrollable mode of $A$ is $\lambda^{*}=-8 / 3<0$ for all values of $c$.

Thus, the system pair $(A, B)$ is stabilizable since we can easily find a gain matrix

$$
K=\left[\begin{array}{ll}
K_{1} & 0
\end{array}\right]=\left[\begin{array}{lll}
k_{1} & k_{2} & 0
\end{array}\right]
$$

so that $A_{1}+B_{1} K_{1}$ is Hurwitz. Thus, the assumption (H2) of Theorem 1 also holds.

\subsection{The Constant Tracking Problem for $x_{1}$}

Here, the tracking problem for the simplified Lorenz chaotic system (7) is given by

$$
\begin{aligned}
& \dot{x}_{1}=10\left(x_{2}-x_{1}\right) \\
& \dot{x}_{2}=-x_{1} x_{3}+(24-4 c) x_{1}+c x_{2}+u \\
& \dot{x}_{3}=x_{1} x_{2}-\frac{8}{3} x_{3} \\
& e=x_{1}-\omega
\end{aligned}
$$

By Theorem 1, the regulator equations of the system (9) are obtained as

$$
\begin{aligned}
10\left[\pi_{2}(\omega)-\pi_{1}(\omega)\right] & =0 \\
-\pi_{1}(\omega) \pi_{3}(\omega)+(24-4 c) \pi_{1}(\omega)+c \pi_{2}(\omega)+\varphi(\omega) & =0 \\
\pi_{1}(\omega) \pi_{2}(\omega)-\frac{8}{3} \pi_{3}(\omega) & =0 \\
\pi_{1}(\omega)-\omega & =0
\end{aligned}
$$


International Journal of Control Theory and Computer Modelling (IJCTCM) Vol.1, No.3, November 2011

Solving the regulator equations (10) for the system (9), we obtain the unique solution as

$$
\begin{aligned}
& \pi_{1}(\omega)=\omega \\
& \pi_{2}(\omega)=\omega \\
& \pi_{3}(\omega)=\frac{3 \omega^{2}}{8} \\
& \varphi(\omega)=3 \omega\left[\frac{\omega^{2}}{8}+c-8\right]
\end{aligned}
$$

Using Theorem 1 and the solution (11) of the regulator equations for the system (9), we obtain the following result which provides a solution of the output regulation problem for (9).

Theorem 2. A state feedback control law solving the output regulation problem for the simplified Lorenz chaotic system (9) is given by

$$
u=\varphi(\omega)+K[x-\pi(\omega)]
$$

where $\varphi(\omega), \pi(\omega)$ are defined as in (11) and the gain matrix $K$ is given by

$$
K=\left[\begin{array}{ll}
K_{1} & 0
\end{array}\right]
$$

with $K_{1}$ chosen so that $A_{1}+B_{1} K_{1}$ is Hurwitz.

\subsection{The constant Tracking Problem for $x_{2}$}

Here, the tracking problem for the unified chaotic system (7) is given by

$$
\begin{aligned}
& \dot{x}_{1}=10\left(x_{2}-x_{1}\right) \\
& \dot{x}_{2}=-x_{1} x_{3}+(24-4 c) x_{1}+c x_{2}+u \\
& \dot{x}_{3}=x_{1} x_{2}-\frac{8}{3} x_{3} \\
& e=x_{2}-\omega
\end{aligned}
$$

By Theorem 1, the regulator equations of the system (13) are obtained as

$$
\begin{array}{r}
10\left[\pi_{2}(\omega)-\pi_{1}(\omega)\right]=0 \\
-\pi_{1}(\omega) \pi_{3}(\omega)+(24-4 c) \pi_{1}(\omega)+c \pi_{2}(\omega)+\varphi(\omega)=0 \\
\pi_{1}(\omega) \pi_{2}(\omega)-\frac{8}{3} \pi_{3}(\omega)=0 \\
\pi_{2}(\omega)-\omega=0
\end{array}
$$

Solving the regulator equations (14) for the system (13), we obtain the unique solution as 
International Journal of Control Theory and Computer Modelling (IJCTCM) Vol.1, No.3, November 2011

$$
\begin{aligned}
& \pi_{1}(\omega)=\omega \\
& \pi_{2}(\omega)=\omega \\
& \pi_{3}(\omega)=\frac{3 \omega^{2}}{8} \\
& \varphi(\omega)=3 \omega\left[\frac{\omega^{2}}{8}+c-8\right]
\end{aligned}
$$

Using Theorem 1 and the solution (15) of the regulator equations for the system (13), we obtain the following result which provides a solution of the output regulation problem for (13).

Theorem 3. A state feedback control law solving the output regulation problem for the unified chaotic system (13) is given by

$$
u=\varphi(\omega)+K[x-\pi(\omega)],
$$

where $\varphi(\omega), \pi(\omega)$ are defined as in (15) and the gain matrix $K$ is given by

$$
K=\left[\begin{array}{ll}
K_{1} & 0
\end{array}\right]
$$

with $K_{1}$ chosen so that $A_{1}+B_{1} K_{1}$ is Hurwitz.

\subsection{The constant Tracking Problem for $x_{3}$}

Here, the tracking problem for the unified chaotic system (7) is given by

$$
\begin{aligned}
& \dot{x}_{1}=10\left(x_{2}-x_{1}\right) \\
& \dot{x}_{2}=-x_{1} x_{3}+(24-4 c) x_{1}+c x_{2}+u \\
& \dot{x}_{3}=x_{1} x_{2}-\frac{8}{3} x_{3} \\
& e=x_{3}-\omega
\end{aligned}
$$

By Theorem 1, the regulator equations of the system (17) are obtained as

$$
\begin{aligned}
10\left[\pi_{2}(\omega)-\pi_{1}(\omega)\right] & =0 \\
-\pi_{1}(\omega) \pi_{3}(\omega)+(24-4 c) \pi_{1}(\omega)+c \pi_{2}(\omega)+\varphi(\omega) & =0 \\
\pi_{1}(\omega) \pi_{2}(\omega)-\frac{8}{3} \pi_{3}(\omega) & =0 \\
\pi_{3}(\omega)-\omega & =0
\end{aligned}
$$

Solving the regulator equations (18) for the system (17), we obtain the unique solution as 


$$
\begin{aligned}
& \pi_{1}(\omega)=\sqrt{\frac{8 \omega}{3}} \\
& \pi_{2}(\omega)=\sqrt{\frac{8 \omega}{3}} \\
& \pi_{3}(\omega)=\omega \\
& \varphi(\omega)=\sqrt{\frac{8 \omega}{3}}[\omega+3 c-24]
\end{aligned}
$$

Using Theorem 1 and the solution (19) of the regulator equations for the system (17), we obtain the following result which provides a solution of the output regulation problem for (17).

Theorem 4. A state feedback control law solving the output regulation problem for the unified chaotic system (17) is given by

$$
u=\varphi(\omega)+K[x-\pi(\omega)]
$$

where $\varphi(\omega), \pi(\omega)$ are defined as in (15) and the gain matrix $K$ is given by

$$
K=\left[\begin{array}{ll}
K_{1} & 0
\end{array}\right]
$$

with $K_{1}$ chosen so that $A_{1}+B_{1} K_{1}$ is Hurwitz.

\section{NUMERICAL SimULATIONS}

We consider the constant reference signal as $\omega=2$.

Also, we take $c=4$ in the simplified Lorenz system (7).

For achieving internal stability of the state feedback regulator problem, a feedback gain matrix $K$ must be chosen so that $A+B K$ is Hurwitz.

As noted in Section 3, $\lambda^{*}=-8 / 3$ is always a stable eigenvalue of $A+B K$.

Suppose we wish to choose a gain matrix such that the other two eigenvalues of $A+B K$ are $\{-5,-5\}$. A simple calculation using Ackermann's formula [20] gives $K_{1}=\left[\begin{array}{ll}-10.5 & -4\end{array}\right]$.

Thus, it follows that $K=\left[\begin{array}{ll}K_{1} & 0\end{array}\right]=\left[\begin{array}{lll}-10.5 & -4 & 0\end{array}\right]$.

For the numerical simulations, the fourth order Runge-Kutta method with step-size $h=10^{-6}$ is deployed to solve the systems of differential equations using MATLAB.

\subsection{Constant Tracking Problem for $x_{1}$}

Suppose that the initial conditions are taken as

$$
x_{1}(0)=10, x_{2}(0)=14, x_{3}(0)=9 .
$$

The simulation graph is depicted in Figure 2 from which it is clear that the state trajectory $x_{1}(t)$

tracks the constant reference signal $\omega=2$ in 2 seconds. 
International Journal of Control Theory and Computer Modelling (IJCTCM) Vol.1, No.3, November 2011

\subsection{Constant Tracking Problem for $x_{2}$}

Suppose that the initial conditions are taken as

$$
x_{1}(0)=22, x_{2}(0)=19, x_{3}(0)=12 .
$$

The simulation graph is depicted in Figure 3 from which it is clear that the state trajectory $x_{2}(t)$ tracks the constant reference signal $\omega=2$ in 2 seconds.

\subsection{Constant Tracking Problem for $x_{3}$}

Suppose that the initial conditions are taken as

$$
x_{1}(0)=6, \quad x_{2}(0)=10, \quad x_{3}(0)=21 .
$$

The simulation graph is depicted in Figure 4 from which it is clear that the state trajectory $x_{3}(t)$ tracks the constant reference signal $\omega=2$ in 2 seconds.

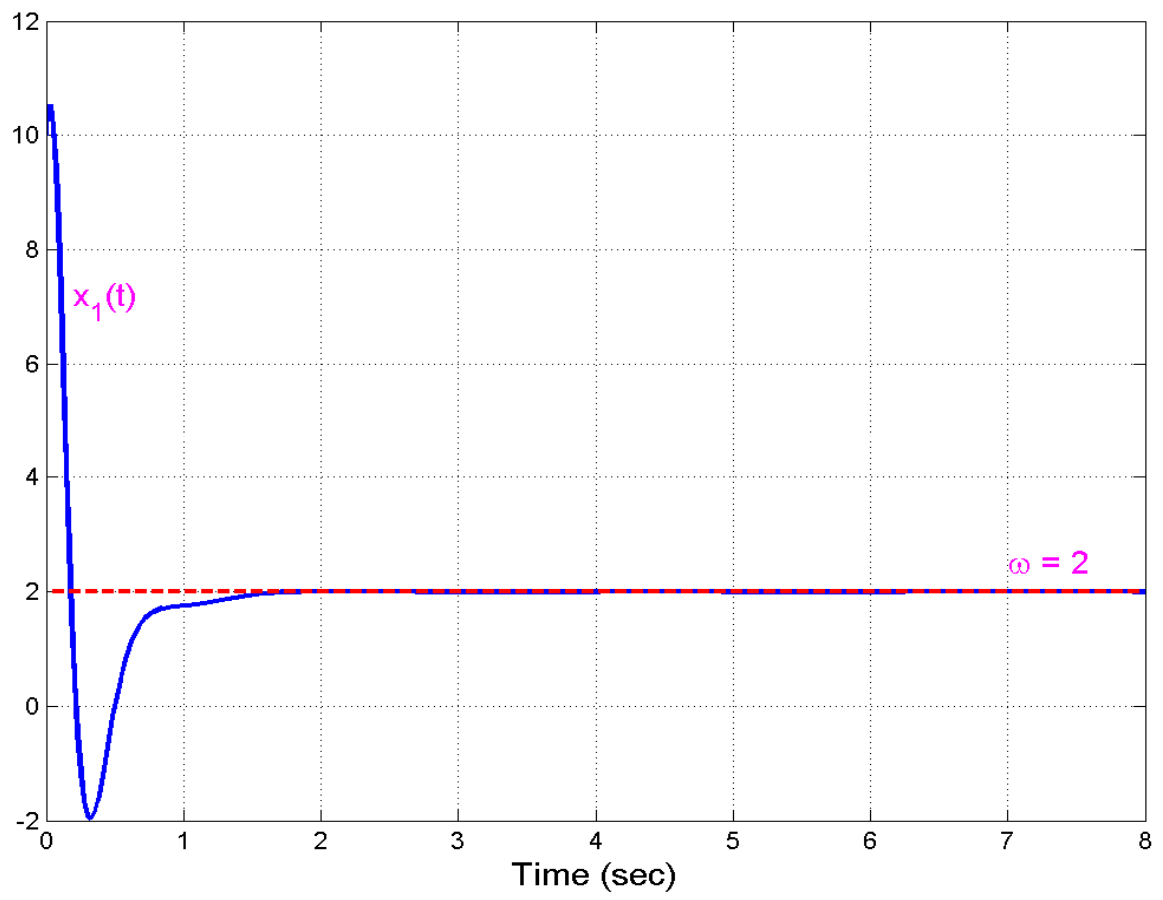

Figure 2. Constant Tracking Problem for $x_{1}$ 
International Journal of Control Theory and Computer Modelling (IJCTCM) Vol.1, No.3, November 2011

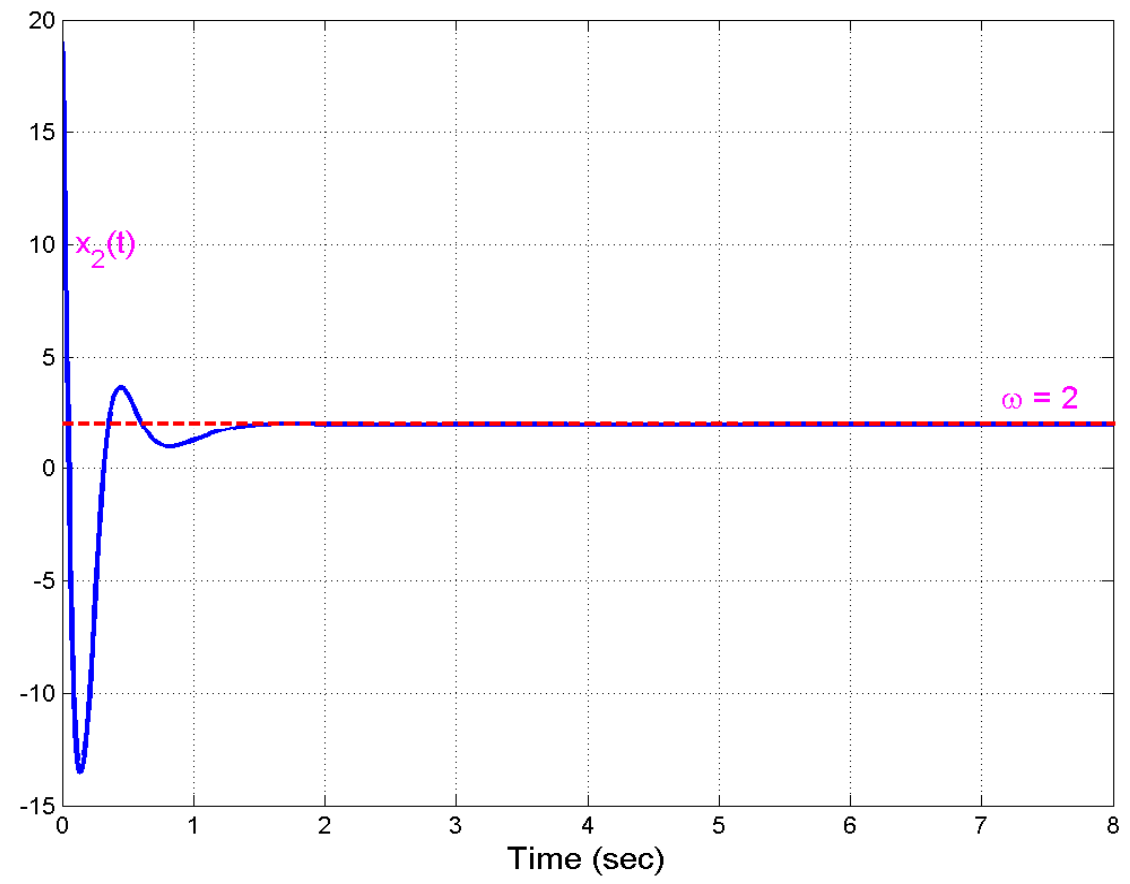

Figure 3. Constant Tracking Problem for $x_{2}$

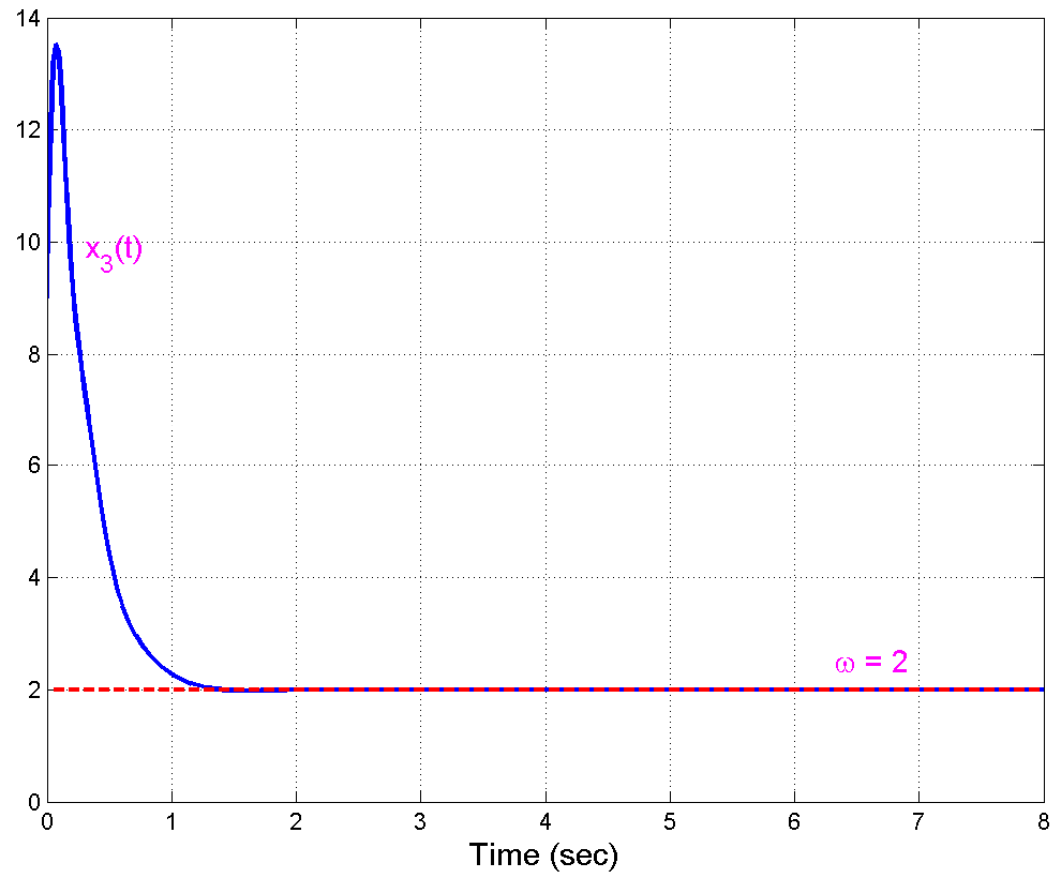

Figure 4. Constant Tracking Problem for $x_{3}$ 


\section{Conclusions}

In this paper, the output regulation problem for the simplified Lorenz chaotic system (2009) has been studied in detail and a complete solution for the output regulation problem for the simplified Lorenz chaotic system has been presented as well for the tracking of constant reference signals. Explicitly, using the regulator equations (Byrnes and Isidori, 1990), state feedback control laws for regulating the output of the simplified Lorenz system have been derived. Numerical simulation results have been discussed to validate and demonstrate the effectiveness of the regulation results derived in this paper for the simplified Lorenz system.

\section{REFERENCES}

[1] Francis, B.A. \& Wonham, W.M. (1975) "The internal model principle for linear multivariable regulators”, J. Applied Math. Optimization, Vol. 2, pp 170-194.

[2] Byrnes, C.I. \& Isidori, A. (1990) "Output regulation of nonlinear systems", IEEE Trans. Automatic Control, Vol. 35, pp 131-140.

[3] Carr, J. (1981) Applications of Centre Manifold Theory, Springer Verlag, New York.

[4] Mahmoud, N.A. \& Khalil, H.K. (1996) "Asymptotic regulation of minimum phase nonlinear systems using output feedback", IEEE Trans. Automat. Control, Vol. 41, pp 1402-1412.

[5] Fridman, E. (2003) "Output regulation of nonlinear control systems with delay", Systems \& Control Lett., Vol. 50, pp 81-93.

[6] Chen, Z. \& Huang, J. (2005) "Robust output regulation with nonlinear exosystems", Automatica, Vol. 41, pp 1447-1454.

[7] Chen, Z. \& Huang, J. (2005) "Global robust output regulation for output feedback systems", IEEE Trans. Automat. Control, Vol. 50, pp 117-121.

[8] Liu, L. \& Huang, J. (2008) "Global robust output regulation of lower triangular systems with unknown control direction”, Automatica, Vol. 44, pp. 1278-1284.

[9] Immonen, E. (2007) "Practical output regulation for bounded linear infinite-dimensional state space systems", Automatica, Vol. 43, pp 786-794.

[10] Pavlov, A., Van de Wouw, N. \& Nijmeijer, H. (2007) "Global nonlinear output regulation: convergence based controller design”, Automatica, Vol. 43, pp 456-463.

[11] Xi, Z. \& Ding, Z. (2007) "Global robust output regulation of a class of nonlinear systems with nonlinear exosystems”, Automatica, Vol. 43, pp 143-149.

[12] Serrani, A. \& Isidori, A. (2000) "Global robust output regulation for a class of nonlinear systems", Systems \& Control Letters, Vol. 39, pp 133-139.

[13] Serrani, A., Isidori, A. \& Marconi, L. (2000) "Semiglobal output regulation for minimum phase systems", Internat. J. Robust Nonlinear Control, Vol. 10, pp 379-396.

[14] Marconi, L., Isidori, A. \& Serrani, A. (2004) "Non-resonance conditions for uniform observability in the problem of nonlinear output regulation", Systems \& Control Letters, Vol. 53, pp 281-298.

[15] Sundarapandian, V. (2011) "New results on the output regulation of the modified Arneodo system by state feedback control", Advanced Computing: An International Journal, Vol. 2, No. 2, pp 1930 .

[16] Sundarapandian, V. (2011) "Output regulation of the Shimizu-Morioka system by state feedback control," International Journal of Advanced Information Technology, Vol. 1, No. 2, pp 1-10.

[17] Sundarapandian, V. (2011) “Output regulation of the Wang system”, International Journal of Computer Information Services, Vol. 2, No. 3, pp 36-41. 
International Journal of Control Theory and Computer Modelling (IJCTCM) Vol.1, No.3, November 2011

[18] Lü, J., Chen, G., Cheng, D.Z. \& Celikovsky, S. (2002) "Bridge the gap between the Lorenz system and the Chen system", Internat. J. Bifurcation and Chaos, Vol. 12, pp. 2917-2926.

[19] Lorenz, E.N. (1963) "Deterministic nonperiodic flow”, J. Atmos. Science, Vol. 20, pp. 130-141.

[20] Ogata, K. (1997) Modern Control Engineering, Prentice Hall, New Jersey, U.S.A.

\section{Author}

Dr. V. Sundarapandian obtained his Doctor of Science degree in Electrical and Systems Engineering from Washington University, Saint Louis, USA under the guidance of Late Dr. Christopher I. Byrnes (Dean, School of Engineering and Applied Science) in 1996. He is currently Professor in the Research and Development Centre at Vel Tech Dr. RR \& Dr. SR Technical University, Chennai, Tamil Nadu, India. He has published over 170 refereed international publications. He has published over 90 papers in National Conferences and over 50 papers in International Conferences. He is the Editor-in-Chief of International Journal of Mathematics and Scientific Computing, International Journal of Instrumentation and

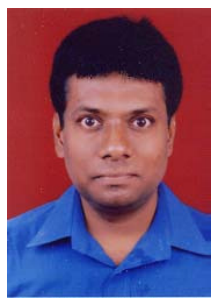
Control Systems, International Journal of Control Systems and Computer Modelling, etc. His research interests are Linear and Nonlinear Control Systems, Chaos Theory and Control, Soft Computing, Optimal Control, Process Control, Operations Research, Mathematical Modelling, Scientific Computing using MATLAB etc. He has delivered several Key Note Lectures on Linear and Nonlinear Control Systems, Chaos Theory and Control, Scientific Computing using MATLAB/SCILAB, etc. 